\title{
SIMULATION OF MULTIPLE STEADY STATES IN A POROUS CATALYST DUE TO PHASE TRANSITION
}

\author{
Dong Hyun KIM and Young Gul KIM \\ Department of Chemical Engineering, \\ Korea Advanced Institute of Science, \\ P.O. Box 150, Chongyangni, Seoul, Korea
}

\begin{abstract}
From numerical calculations, two of the four multiple steady states (branches $\mathrm{A}$ and $\mathrm{C}$ ) obtained on a biporous $\mathrm{Pt} / \mathrm{AI}_{2} \mathrm{O}_{3}$ catalyst surrounded by reacting mixture containing saturated vapor of cyclohexene reported in the preceding paper $^{\hat{\theta}}$ can be attributed to the behavior of the catalyst filled with vapor mixture and condensed liquid mixture in its micropores. Stability analysis based on heat generation and heat removal curves without considering phase change in the catalyst interior showed only a single steady state, i.e. the rate of vapor-filled catalyst, while incorporating the effect of phase change on the heat generation curve yielded two steady states which gave good agreement with those of experiments within experimental error.
\end{abstract}

\section{Introduction}

The intent of the present study is to portray closely the physicochemical situations and the intriguing behavioral characteristics of the reaction rate presented in the preceding paper ${ }^{6}$. The major thrust is toward rate calculations of a single catalyst pellet which has vapor or condensed liquid in its pores, based on stability analysis incorporating the effect on reaction rate of the changes in effective diffusivity and intrinsic kinetics with phase change in pores. However, systematic studies on such matters as how much those quantities will change with condensation in pores have not yet been reported. The degree of condensation in the catalyst interior depends on the temperature difference between the catalyst and input reactant stream and as pointed out in the preceding paper the functional relationship between these two quantities is uncertain. In this work these quantities, effective diffusivity, intrinsic reaction rate and degree of condensation, are estimated with approximations on the catalyst internal state. Much study on this aspect remains to be done.

\section{Development of Calculation Scheme}

The basis of calculation contains as external problems that heat and mass transport, and axial and radial dispersion of each transport, and as internal problems the pore diffusion, the degree of condensation and the intrinsic kinetics.

On the external problems, Wicke ${ }^{15 t}$ described these

Received December 15, 1980. Correspondence concerning this article should be addressed to Y. G. Kim. processes with measurements of temperature and concentration profiles along short combustion zones in packed beds and showed that the axial dispersion of mass is very small under the condition of small Reynolds number, and that there is appreciable backmixing of heat against the gas flow. These observations clearly indicate that the concentrations of the bulk surrounding a single catalyst pellet imbedded in a large amount of inert particles can be safely assumed to be the same as those of the feed stream. However, the bulk temperature in this case may be appreciably higher than that of the feed stream because of the backmixing of reaction heat, and may vary in relation to the catalyst surface temperature. To calculate the catalyst surface temperature with an equation such as

$$
\text { rate }(-\Delta H)=h\left(T_{s}-T_{o}\right)
$$

the bulk temperature $T_{o}$ should be estimated beforehand. However, quantitative estimation under such a steep temperature gradient is nearly impossible in view of the inadequacy of knowledge of heat transfer. But this difficulty can be circumvented and a pseudo bulk temperature, i.e. feed stream temperature, can be used if a heat transfer relation measured under the influences of significant axial and radial heat conduction and based on the temperature differences between feed stream and heat source is employed. The heat transfer correlation derived in the preceding paper $\left.^{6}\right)$ seems to meet these requirements well.

$$
\varepsilon j_{h}=4.92 R e_{p}^{-0.755}
$$

For gas-phase mass transfer in packed beds, Petrovic and Thodos $^{11)}$ correlated mass transfer data as 


$$
\varepsilon j_{d}=0.357 R e_{p}^{-0.359}
$$

Using these two correlations (2) and (3), the bulk conditions can be regarded as those of the feed stream in calculations of surface conditions.

To simplify development of modeling equations on heat generation in the catalyst the following assumptions are necessary:

1. Catalyst surface condition is uniform.

2. Isothermal condition is maintained inside the catalyst.

3. Mass transport in the catalyst can be described by Fick's law with a constant diffusivity.

4. The limiting reactant in the catalyst is cyclohexene.

5. The mass balance in the catalyst can be described by the balance equation for a slab catalyst using a proper characteristic length as recommended by various investigators ${ }^{1,2,10}$ for large values of Thiele modulus and complex geometries.

6. Nearly all the internal active surface lies in the micropores of the catalyst.

7. The solubilization rate of hydrogen at phase boundaries in liquid-filled pores is very rapid.

For convenience each component in the reaction mixture is subscripted: 1 for hydrogen, 2 for cyclohexene, 3 for cyclohexane, and 4 for nitrogen. The mass and heat transport rates are related by

$$
h\left(T_{s}-T_{0}\right)=(-\Delta H) k_{2}\left(P_{2 o}-P_{s}\right)
$$

the transport rates of each component are related by

$$
k_{2}\left(P_{2 o}-P_{2 s}\right)=k_{1}\left(P_{10}-P_{1 s}\right)=-k_{3}\left(P_{3 o}-P_{3 s}\right)
$$

Using mass and heat transfer Colburn $j$ factors the relation between cyclohexene partial pressure difference and the temperature difference is given as

$$
P_{2 o}-P_{2 s}=\frac{j_{h} C_{p} \bar{M} P_{f 2}}{j_{d}(-\Delta H)}\left(\frac{S c_{2}}{P r}\right)^{2 / 3}\left(T_{s}-T_{o}\right)
$$

With this cyclohexene partial pressure difference the partial pressure difference of each component can be written as follows:

$$
\begin{aligned}
& P_{10}-P_{1 s}=\frac{P_{f 1}}{P_{f 2}}\left(\frac{S c_{1}}{S c_{2}}\right)^{2 / 3} \\
& P_{3 s}-P_{30}=\frac{P_{f 3}}{P_{f 2}}\left(\frac{S c_{3}}{S c_{2}}\right)^{2 / 3}\left(P_{20}-P_{2 s}\right) \\
& P_{4 s}=P_{t}-\left(P_{1 s}+P_{2 s}+P_{3 s}\right)
\end{aligned}
$$

The catalyst surface conditions have now been obtained as functions of the temperature difference or its related partial pressure difference, and with this information the reaction rate or the heat generated as a result of the reaction can be readily calculated. It should be noted here that the intrinsic reaction rate and the effective diffusivity values are affected not only by the surface conditions but also by the degree of condensation, which is also a function of the surface condition, namely the temperature difference between the surface and the bulk stream. Unfortunately, nothing on this aspect has been reported in the literature. However, to go further in this discussion and to avoid complicated and unnecessary details in rate calculations the interior states of the catalyst are simply divided into state i) in which all the pores in the catalyst are filled with a vapor mixture, and state ii) in which all the micropores are filled with a condensed liquid while the macropores are still filled with the vapor mixture. This binary classification allows a simple interpretation of changes in effective diffusivity as proposed by the well-known random pore model $^{14)}$, and of changes in intrinsic kinetics.

If the micropores are previously filled with condensed liquid, the liquid contained in them does not seem to evaporate appreciably until the temperature difference between catalyst and feed stream reaches about $7^{\circ} \mathrm{C}$, for which the corresponding pore radius read from Fig. 1 in the preceding paper is $80 \AA$. With regard to the pore size distribution curve the majority of micropores lie in the range of 25 to $80 \AA$ in radius. Therefore, to obtain a fully vapor-filled catalyst with pore sizes extending down to $25 \AA$, the temperature difference should increase up to $20^{\circ} \mathrm{C}$.

Between 20 and $7^{\circ} \mathrm{C}$, the degree of condensation in micropores will be a function of the temperature difference, and in this region estimation of effective diffusivity and the intrinsic reaction rate is difficult because of the absence of information. However, it is apparent in a qualitative sense that the reaction rate will certainly increase in this region. For the condensation in micropores of a vapor-filled catalyst, the estimated range of temperature difference is between 12 and $4^{\circ} \mathrm{C}$. As pointed out previously, these temperature differences are merely indications of the magnitudes to be expected. For a given temperature difference these considerations should be accounted for in the rate calculations according to the direction of change of reaction conditions.

In the following the estimation of parameters, effective diffusivity and intrinsic reaction rate according to the internal states of the catalyst, i.e. state i) and state ii), will be briefly discussed.

State i) According to the random pore model all the diffusion paths, i.e. through macropores, through micropores and through micro-and-macropores in series, participate in diffusion through the catalyst interior when the vapor phase is maintained in it. Hence the effective diffusivity of a reacting species $A$ is expressed as the sum of these contributions.

$$
D_{e A}=\varepsilon_{\alpha}^{2} D_{a}+\left(1-\varepsilon_{a}\right)^{2} D_{i}+4 D_{a}\left(1-\varepsilon_{a}\right)\left(1 / D_{a}+1 / D_{i}\right)^{-1}
$$

where 


$$
\begin{aligned}
& D_{a}=\frac{1}{\left(1-\alpha y_{A}\right) / D_{A}+1 / D_{k A a}} \\
& D_{i}=\frac{\varepsilon_{i}^{2} /\left(1-\varepsilon_{a}\right)^{2}}{\left(1-\alpha y_{A}\right) / D_{A}+1 / D_{k A i}}
\end{aligned}
$$

As for the intrinsic reaction rate, the vapor phase rate should be used. Boundart et al. ${ }^{13)}$ studied the cyclohexene hydrogenation reaction and concluded that the reaction was structure-insensitive as the measured turnover frequency, defined as the average number of molecules of cyclohexene consumed per surface platinum atom per second, was not changed significantly when platinum dispersion changed from 14 to $100 \%$. Their reported data were too meager to permit derivation of a rate equation. Lee ${ }^{7}$ ) also studied the reaction and correlated the results in terms of turnover frequency. If his results are rewritten in a convenient unit using the known properties of the catalyst and the platinum dispersion of the catalyst $(70.2 \%)$ listed in Table 1 of the preceding paper $^{\hat{\theta}}$, we obtain

$$
\begin{aligned}
\text { rate }[\mathrm{mol} / \mathrm{m} l \text {-cat } \cdot \mathrm{sec}] & =k P_{\mathrm{H}_{2}}^{n_{2}} \\
T<313^{\circ} \mathrm{K} ; & k=1.06 \times 10^{11} \exp (-21400 / R T) \\
n & =5.23-0.0151 T \\
T<313^{\circ} \mathrm{K} ; & k=48.7 \exp (-7930 / R T) \\
n & =\frac{1}{2}
\end{aligned}
$$

State ii) In this case all the micropores are filled with a liquid mixture. Consequently, the diffusion rate through micropores and through micro- and macropores in series becomes negligible compared with that through macropores because the diffusivity values of gas or vapor phase are 3 to 4 orders of magnitude greater than that of the liquid phase. Thus the effective diffusivity is simply

$$
D_{e A}=\varepsilon_{\alpha}^{2} D_{a}
$$

Since most of the active areas lie in the region of micropores, and micropores are filled with condensed liquid, the liquid-phase intrinsic reaction rate should be used although the apparent phase seen from outside is still vapor. The liquid-phase hydrogenation of cyclohexene was also studied by Boudart et al. ${ }^{8)}$. They concluded that the liquid-phase reaction was also structure insensitive. If their results are rewritten in convenient units in rate calculations

$$
\begin{aligned}
& \text { rate }[\mathrm{mol} / \mathrm{m} l \text {-cat } \cdot \mathrm{sec}]=k P_{\mathrm{H}_{2}} \\
& k=0.220 \exp (-6760 / R T)
\end{aligned}
$$

In actual pore systems having bimodal pore size distribution, it is believed that micropores are interconnected by macropores and too small in size to consider variations in reactant concentration and temperature inside them. Hence the catalyst pellet maintained in state ii) and under the reaction condition can be thought of as a homogeneous system with parameter values calculated by Eqs. (14) and (15).
Since the cyclohexene hydrogenation reaction rate is dependent only on hydrogen partial pressure and temperature, the hydrogen mass balance alone is sufficient in describing the catalyst interior. The dimensionless mass balance in a slab geometry is

$$
\frac{d^{2} y}{d x^{2}}-L \frac{R T k P_{1 s}^{n-1}}{D_{e 1}}-y^{n}=0
$$

Boundary conditions are

$$
\begin{gathered}
0<X<1, \quad P_{2}=0, \quad \frac{d y}{d x}=0 \\
x=1, \quad y=1
\end{gathered}
$$

If $q=d y / d x$, then

$$
q \frac{d q}{d x}-\frac{L^{2} R T k P_{1 s}^{n-1}}{D_{e 1}} y^{n}=0
$$

The hydrogen partial pressure $P_{1}$ within the catalyst in terms of the known $P_{2}$ is

$$
P_{1}=P_{1 s}-\frac{D_{e 2}}{D_{e 1}}\left(P_{2 s}-P_{2}\right)
$$

where

$$
\frac{D_{e 2}}{D_{e 1}}=\left(\frac{M_{2}}{M_{2}}\right)^{1 / 2}
$$

This relation has been proved theoretically and experimentally in porous media and is assumed here to be valid even in a partially liquid-filled catalyst.

The boundary condition of Eq. (19) is

$$
q=0 \text { at } y=y_{0}=1-\left(\frac{M_{1}}{M_{2}}\right)^{1 / 2} \frac{P_{2 s}}{P_{1 s}}
$$

Then the reaction rate is

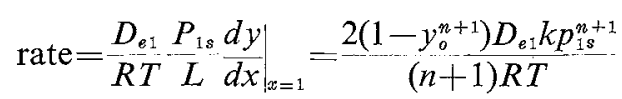

and heat generated is

$$
(-\Delta H)^{2\left(1-y_{0}^{n+1}\right) D_{e 1} k P_{1 s}^{n+1}}
$$

By increasing the temperature difference in small increments the complete heat generation curve can be constructed and the rates are calculated as intersections of the heat generation curve and the heat removal curves.

\section{Results and Discussion}

Rates thus calculated are slightly higher than the experimental values of high-activity catalyst, but the trend with respect to hydrogen partial pressure is similar. Compared to vast differences in predicted and experimental values reported ${ }^{4.9}$, such small deviations of about $8 \%$ are very promising. It could be meaningful to correct the parameter values that give agreement between calculated and experimental rates for each catalyst activity. From the reaction rate expression, Eq. (23), it may be noted that the $\%$ change in value of $D_{e 1}$ or $k$ has the same effect on the reaction rate. As a 


\begin{tabular}{|c|c|c|c|}
\hline \multirow{2}{*}{$\begin{array}{l}\text { Table } \\
\text { Activity }\end{array}$} & \multicolumn{3}{|c|}{ Correction of parameter values } \\
\hline & {$[\mathrm{mol} / \mathrm{g}-\mathrm{cat} \cdot \mathrm{hr}]$} & \multicolumn{2}{|c|}{  } \\
\hline high & 0.102 & 0 & 15.4 \\
\hline medium & 0.090 & 64.0 & 15.4 \\
\hline low & 0.0817 & 77.5 & 15.4 \\
\hline
\end{tabular}

means of correction, all the deviations are assumed to originate solely from the variations in catalyst activity and an uncertainty in effective diffusivity. This is made at a chosen condition, $30^{\circ} \mathrm{C}$ and $327 \mathrm{mmHg} P_{\mathrm{H}_{2}}$, at which the rate data are abundant. For each catalyst activity, the reaction rates and corrected parameter values are listed in Table 1. The calculated rates with corrected parameter values at each reaction temperature show good agreement and are shown in Fig. 1.

The steady state multiplicity which is presumed to result from the coupling of the transport and reaction processes as usually discussed in stability analysi $\mathrm{s}^{3,5,12)}$ is not observed if we do not consider phase change in the catalyst interior. Nor do we observe the sigmoidal characteristics of the heat generation curve which is essential to produce steady state multiplicity without phase change. In Fig. 2, typical heat generation curve and heat removal curve with and without phase changes are shown.

The values of Thiele modulus are always larger than 10 , hence assumption 5 is always satisfied. The assumption that cyclohexene is the limiting reactant and that it is completely consumed is also justified because of the large values of Thiele modulus and greater molar flux of hydrogen than that of cyclohexene (6.5 times) unless the hydrogen partial pressure in the reactant mixture is too small, i.e. less than $20 \mathrm{mmHg}$.

The effect of flow rate on reaction rate of the vaporfilled catalyst is simulated and compared with experimental data in Fig. 3. The quite marked increase in reaction rate and catalyst temperature with increasing flow rate clearly shows that the external mass transfer has more profound influence than the external heat transport on reaction rate. Catalyst surface temperature rise with increasing flow rate was in fact observed in the course of changing reaction conditions, but unfortunately not enough of these data were obtained to compare with the simulated results.

Due to the absence of information on catalyst containing condensed liquid in part of its micropores, an attempt to simulate the rate data corresponding to branch B did not succeed.

However, the calculated results provide confirmation of the expection that the interior state of the catalyst exhibiting the rates of branch $C$ in Figs. 8 and 9 in the previous paper $^{6}$ corresponds to state ii), and Figs. 4 and $\mathbf{5}$ show the anticipated results. For the mediumactivity catalyst the agreement is somewhat poor due to

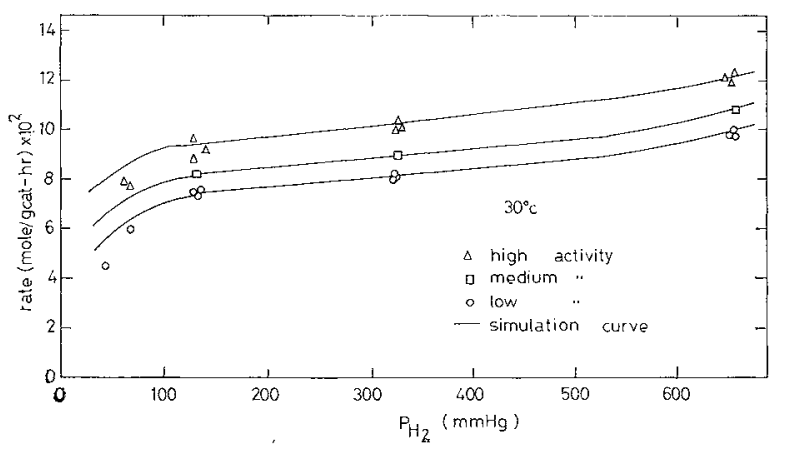

Fig. 1 Comparison of calculated results and experimental data $\left(30^{\circ} \mathrm{C}\right)$



Fig. 2 Shape of heat generation curves (Schematic)

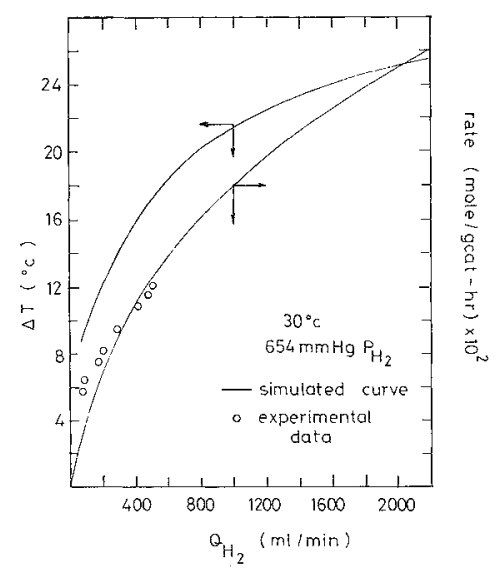

Fig. 3 Dependence of reaction rate and temperature difference on hydrogen flow rate

the scarcity of experimental data. Here branch B can be interpreted in two ways. The first is that a part of the micropores in the catalyst is filled with condensed liquid in view of the fact that branches $\mathrm{A}$ and $\mathrm{C}$ correspond to state i) and state ii) respectively. The second is that, since two catalyst pellets were employed in each of these measurements, i.e. sample $\mathrm{Cl}$ for the lowactivity experiments and $\mathrm{C} 10$ for medium activity, and since the rates of branch $\mathrm{B}$ can be approximated as the mean of the rates of branch $A$ and branch $C$ in the region of the reaction condition where the data of the two branches coexist, in each of the two pellets state i) and state ii) are maintained respectively. Because of the limited experimental data it is uncertain as to which of these two interpretations is correct. More experiments on this aspect would be desirable to determine 


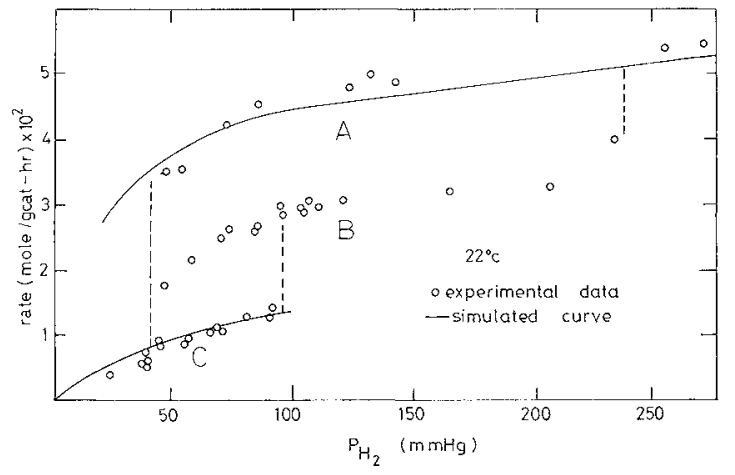

Fig. 4 Comparison of calculated results and experimental data: multiple steady states due to condensation in micropores (low activity)

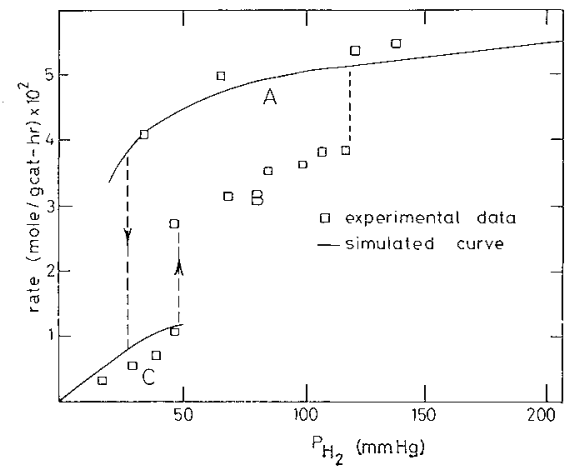

Fig. 5 Comparison of calculated results and experimental data: multiple steady states in micropores (medium activity)

the interior state of the catalyst which exhibited the rate of branch $\mathrm{B}$.

\section{Conclusion}

Reaction rates of the catalyst containing vapor or liquid in its micropores calculated by using simplifying assumptions and available correlations are in good agreement with experimental values reported in the preceding paper, thereby providing a confirmation that the multiple steady states originated from the phase change in catalyst interior.

The dependence of values such as effective diffusivity, intrinsic reaction rate with respect to degree of condensation in porous media and steady state multiplicity on this aspect have not been reported to date, and this work is in itself not complete. There remains much to be done for better understanding of the results of this work and also others to follow that are associated with phase transition in porous catalyst.

$\begin{array}{llr}\text { Nomenclature } & \\ C_{p} & =\text { heat capacity } & {\left[\mathrm{cal} / \mathrm{g} \cdot{ }^{\circ} \mathrm{K}\right]} \\ D_{A} & =\text { bulk diffusivity of component A } & {\left[\mathrm{cm}^{2} / \mathrm{sec}\right]} \\ D_{a} & =\text { composite diffusivity association with } & \\ & \text { macropore } & {\left[\mathrm{cm}^{2} / \mathrm{sec}\right]} \\ D_{e} & =\text { effective diffusivity } & {\left[\mathrm{cm}^{2} / \mathrm{sec}\right]}\end{array}$

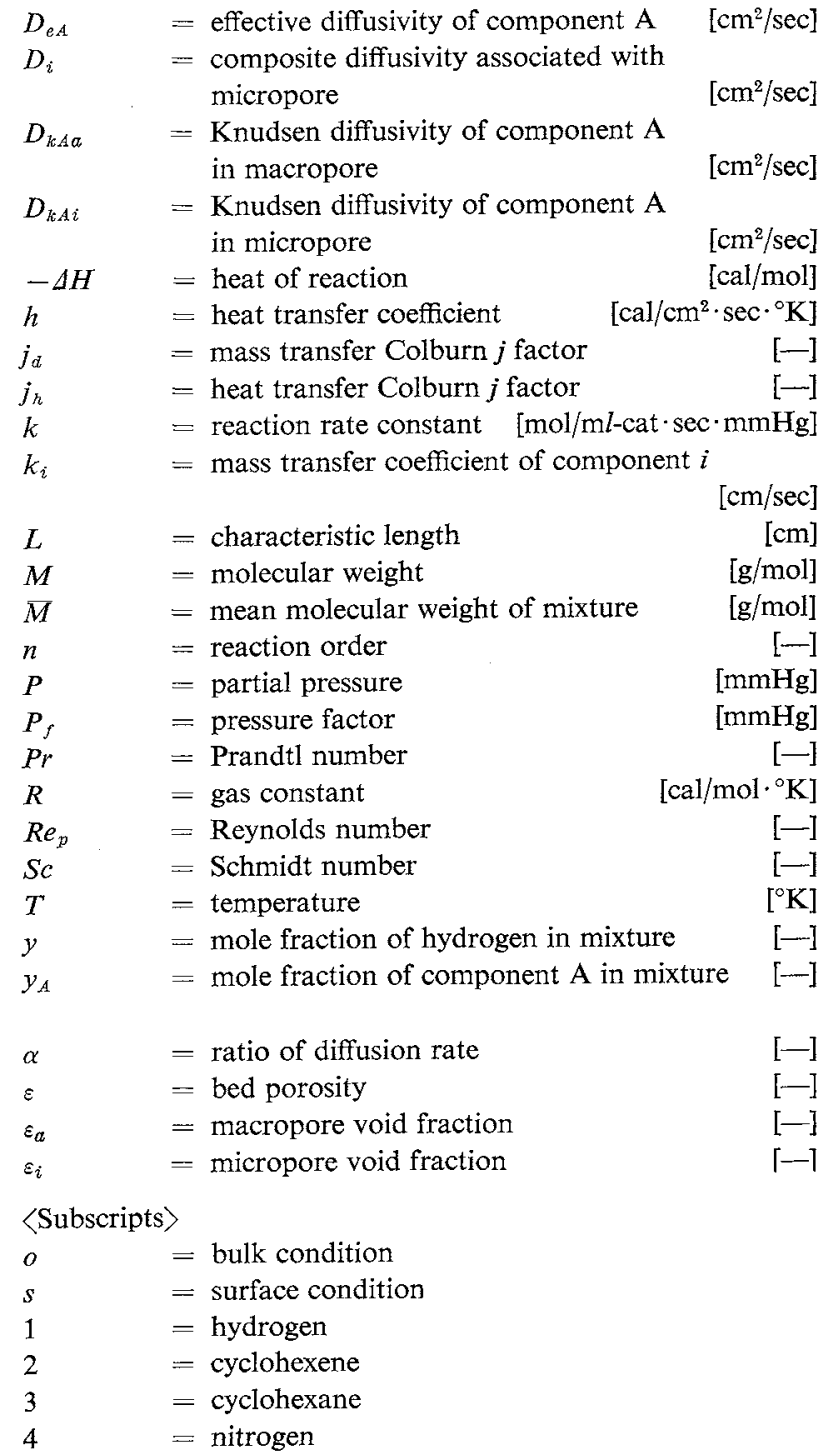

\section{Literature Cited}

1) Aris, R.: Ind. Eng. Chem., Fundam., 4, 227 (1965).

2) Bischoff, K. B.: AIChE J., 11, 351 (1965).

3) Bosch, B. V. D. and D. Luss: Chem. Eng. Sci., 32, 203 (1977).

4) Hesse, D.: idem., 32, 413 (1977).

5) Hlavacek, V. and M. Kubicek: ibid., 25, 1537 (1970).

6) Kim, D. H. and Y. G. Kim: J. Chem. Eng. Japan, 14, 311 (1981)

7) Lee, T. J.: M. Sc. Thesis, Korea Advanced Institute of Science, Seoul, Korea (1980).

8) Madon, R. J., J. P. O'Conell and M. Boudart: AIChE J., 24, 904 (1978)

9) Otani, S. and J. M. Smith: J. Catal., 5, 332 (1966).

10) Petersen, E. E.: "Chemical Reaction Analysis", PrenticeHall, Englewood Cliffs, N. J., U.S.A. (1965).

11) Petrovic, L. J. and G. Thodos: Ind. Eng. Chem., Fundam., 7, 274 (1968).

12) Schmitz, R. A.: Adv. in Chem. Ser., 148, 157 (1975).

13) Segal, E., R. J. Madon and M. Boudart: J. Catal., 52, 45 (1978).

14) Wakao, N. and J. M. Smith: Chem. Eng. Sci., 17, 825 (1962).

15) Wicke, E.: Adv. in Chem. Ser., 109, 183 (1972). 